\title{
Pigeons Show Metamemory by Requesting Reduced Working Memory Loads
}

\author{
Sumie Iwasaki ${ }^{*}$, Hika Kuroshima ${ }^{2}$, and Kazuo Fujita ${ }^{2}$ \\ ${ }^{1}$ Research Center for Child Mental Development, Kanazawa University \\ ${ }^{2}$ Graduate School of Letters, Kyoto University \\ *Corresponding author (Email: s.iwasaki623@gmail.com)
}

Citation - Iwasaki, S., Kuroshima, H., \& Fujita, K. (2019). Pigeons show metamemory by requesting reduced working memory loads. Animal Behavior and Cognition, 6(4), 247-253. https://doi.org/10.26451/abc.06.04.04.2019

\begin{abstract}
Even though many studies have demonstrated that pigeons and humans share various properties of memory systems, pigeons have shown only weak evidence of metamemory ability, for example during delayed matching-to-sample. We suspect that this task might be too demanding to allow metamemory processing within pigeons' working memory. Here, we describe our studies in which pigeons have shown metamemory during a task requiring reduced working memory load, a reference memory task. Pigeons solved a simultaneous chaining task, and they were sometimes given the opportunity to ask for "hints" about the next correct response in a sequence before or during the task. Some pigeons' hint-seeking behavior varied according to their reference memory states or knowledge states, suggesting that they used metamemory when engaged in a reduced working memory load task. We propose that metamemory ability is dependent on working memory capacity, and that it is important to use suitable cognitive tasks for evaluate metamemory abilities in diverse species.
\end{abstract}

Keywords - Pigeons, Metamemory, Information-seeking, Working memory loads, Reference memory

Metamemory is a cognitive function that allows monitoring one's own memory state or trace, and thereby enhanced control of behavior. Humans can report or judge their own memory states verbally. These reports correlate significantly with accuracy on memory tasks (Nelson, 1996). Studies using nonverbal procedures have demonstrated metamemory in other mammals (chimpanzees (Pan troglodytes): Call \& Carpenter, 2001; orangutans (Pongo pygmaeus): Suda-King, 2008; rhesus monkeys (Macaca mulatta): Brown, Basile, Templer, \& Hampton, 2019; Hampton, 2001; Smith, Shields, Allendoerfer, \& Washburn, 1998; Templer, Brown, \& Hampton, 2018; rats (Rattus norvegicus): Foote \& Crystal, 2007; Templer, Lee, \& Preston, 2017; Yuki \& Okanoya, 2017).

Inman and Shettleworth (1999) investigated metamemory in pigeons (Columba livia) in a delayed matching-to-sample (DMTS) task. In this task, pigeons were required to peck a sample stimulus at the start of trial. After a delay three comparison stimuli including the sample stimulus were presented. When the pigeons pecked the same stimulus as the sample, they got six pellets (a high reward) for a correct response; however, incorrect responses led to a timeout period and no reward. In some trials, pigeons were given an opportunity to choose between taking a test or escaping it before or during presentation of the comparison stimuli. Choosing the escape option always resulted in them receiving only three pellets (a medium reward level). The pigeons' accuracies were lower in longer-delay trials, during which they frequently escaped the test when this option was presented simultaneously with the comparison stimuli. However, when the escape option was presented before the longer-delay trial comparison stimuli, they 
failed to use it. Sutton \& Shettleworth (2008) also investigated escape responses in pigeons during DMTS. In their first experiment, they confirmed that subjects preferred 12 (high value) to 5 (low value) pellets with higher probabilities for 12 pellets, but their preferences were reversed with lower probabilities for 12 pellets. Then, they used the reward structure (12 pellets for a correct response, 0 pellets for an incorrect response, and 5 pellets for an escape response) in metacognition tests. However, pigeons failed to escape the longer-delay trials before the test appeared, and therefore showed no evidence of metamemory.

Another study using DMTS showed a limited evidence of metamemory in pigeons (Adams \& Santi, 2011). In initial testing, escape responses did not increase in longer-delay trials, but after prolonged training in which pigeons chose to take or to escape a test, they finally learned to escape it more frequently in longer-delayed trials than in shorter-delayed trials. However, the prolonged training could provide leaning opportunities to maximize rewards (e.g., in longer delayed trials, pigeons got more food when they escaped than when they took a test). Therefore, the results of that study cannot rule out the possibility of associative learning between retention intervals and escape responses. Large-billed crows (Corvus macrorhynchos) also failed to show metamemory during a prospective judgment in DMTS (Goto $\&$ Watanabe, 2012). The crows escaped the memory test more frequently in long-retention than shortretention interval trials, but it is conceivable that they used the length of retention interval rather than the strength of their memory trace as a cue to avoid taking the test.

In contrast to the relatively weak evidence of metamemory in pigeons and crows, macaques have shown strong evidence of metacognition during DMTS; they adequately escaped a test before presentation of comparison stimuli when their memory traces were weaker (Brown et al., 2019; Hampton, 2001; Templer et al., 2018). Smith, Couchman, and Beran (2014) concluded that it may be difficult for pigeons to access to their memory trace, compared to monkeys.

Despite the lack of clear evidence for metamemory, various properties of the pigeon memory system are similar to those of humans (e.g., serial position curves: Wright, Santiago, Sands, Kendrick, \& Cook, 1985; chunking: Terrace, 1991). Moreover, pigeons adjust their memory strategies according to conditions (e.g., Maki \& Hegvik, 1980). Pigeons have been reported to monitor their perceptual state in the context of a perceptual discrimination task (Nakamura, Watanabe, Betsuyaku, \& Fujita, 2011) and a same-different discrimination task (Castro \& Wasserman, 2012). Considering these results, it might be too early to conclude that pigeons lack metamemory.

As noted, the DMTS task is commonly used in metamemory studies (e.g., Foote \& Crystal, 2007; Fujita, 2009; Hampton, 2001; Inman \& Shettleworth, 1999; Sutton \& Shettleworth, 2008; Takagi \& Fujita, 2018), but it might not be suitable for pigeons due to its large working memory requirements. During this task, samples changed every trial, so that the subjects had to remember a sample while avoiding proactive interference from previous trials. Subjects had to use some working memory resources to solve the task. Studies with adult humans have shown that overloading working memory with a task interferes with the accuracy of metacognitive judgments (Finley, Benjamin, \& McCarley, 2014; Sannomiya \& Ohtani, 2015). Also, Smith, Coutinho, Church, and Beran (2013) demonstrated that working memory loads and attentional resources of tasks affected monkeys' escape responses in the DMTS task. They found that a concurrent task disrupted the macaques' uncertainty responses, but it did not impact the primary perceptual judgments within which the monitoring of uncertainty occurred. Given these results, we should use a task with a reduced working memory load to better evaluate metamemory in pigeons.

Kornell, Son, and Terrace (2007) investigated hint-seeking behavior in rhesus monkeys during a simultaneous chaining task, a type of reference memory task. This task requires fewer working memory resources, because the same stimuli are presented in all trials, although in different locations. The subjects touched four arbitrary photographs in a predetermined correct order. In half of the trials subjects were given the opportunity to request "hints." When a subject chose a hint-seeking icon on a monitor, the item that it should touch next was highlighted, but this also resulted in delivery of a lower-value reward (a banana pellet) at the end of a correctly completed trial. By contrast, when the subject responded correctly without any hint, it received a high-value reward (an M\&M). Errors were followed by a timeout. The 
results showed that the monkeys requested the hint more often in novel list trials than familiar list trials, and there was a strong negative correlation between hint-seeking rates and accuracy. Thus, the monkeys applied appropriate strategies to compensate for a lack of memory in the reference memory task.

We applied the paradigm used by Kornell et al. (2007) to investigate metamemory in pigeons (Iwasaki, Watanabe, \& Fujita, 2013; see Figure 1). In this study, pigeons were given opportunities to seek a hint which guided a correct response during a simultaneous chaining task. We adjusted the probability of primary reinforcement between $50 \%$ and $100 \%$ for trials in which the hint was requested, but the probability of primary reinforcement was $100 \%$ for trials without a hint. Pigeons' choices for the hint icon were analyzed during a three-item simultaneous chaining task. Two of four subjects sought hints in early sessions more often than in the final sessions during learning of novel sequences, and the frequency of hint-seeking was inversely correlated with accuracy on trials in which hints were unavailable. These results suggested that pigeons were able to monitor their reference memory (or knowledge) state, and thereby control their hint-seeking behavior.

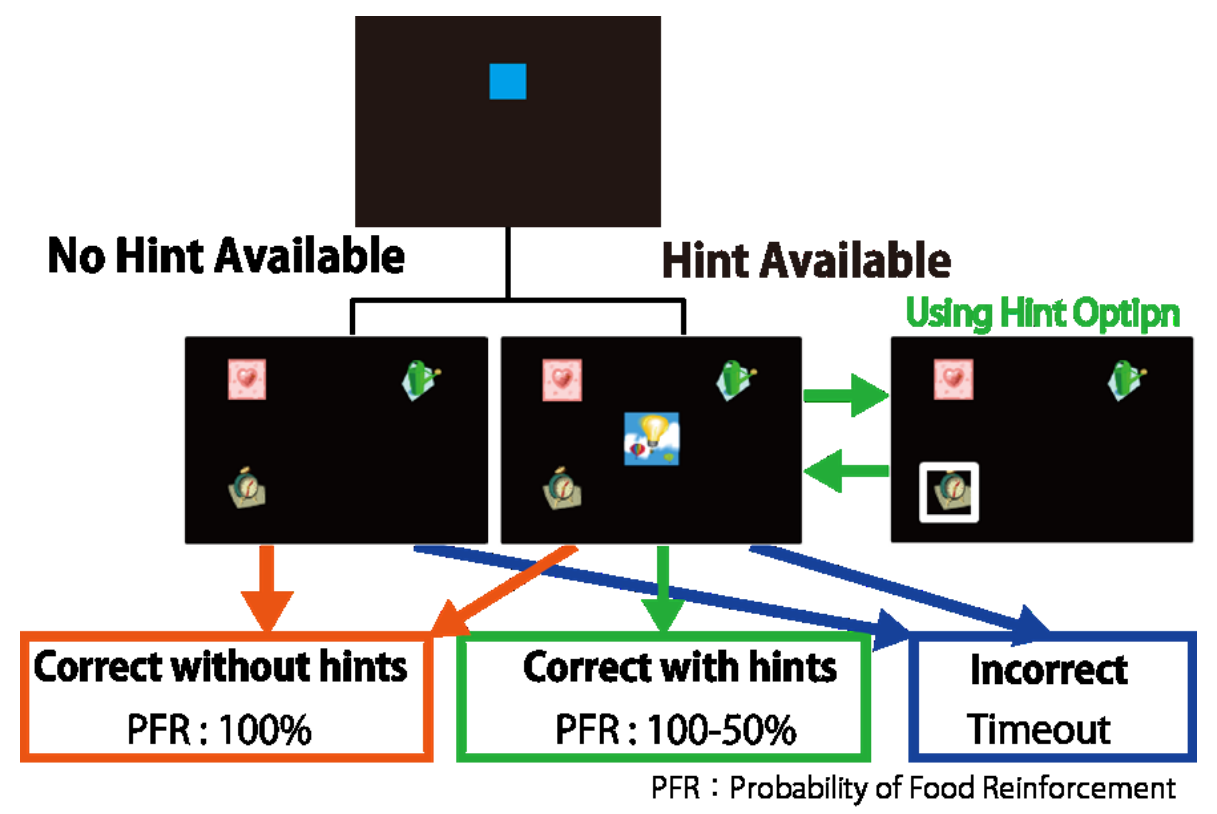

Figure 1. A schematic representation of Iwasaki et al. (2013). After pecking a self-start key, subjects faced solving a three-item simultaneous chaining task. In half of the trials subjects were given to a hint option which showed the next response (Hint Available trials). In other trials, hints were unavailable (No Hint Available trials). All correct responses without hints were reinforced by food, but $50-100 \%$ of correct responses with hint trials were reinforced. Incorrect responses resulted in a timeout irrespective of whether a hint was used. From Iwasaki, S. et al. (2013). Do pigeons (Columba livia) seek information when they have insufficient knowledge? Animal Cognition, 16, 211-221. Copyright 2015 by Springer Nature.

However, our study (Iwasaki et al., 2013) still left open the possibility of a non-metamemory explanation of the birds' behavior: namely, a "response competition" account, proposed by Hampton (2009). In the simultaneous chaining task, the hint-seeking icon was presented along with the test stimuli; the main tasks (requiring a primary response) and the possibility of a hint-seeking response (a secondary "metacognitive" response) might compete with each other in terms of stimulus control due to past outcomes. In this situation, the less likely the primary response is to occur (e.g., on difficult trials), the more likely the secondary response is to occur. The metacognitive-like response could emerge from strength of responses (e.g., how well-rewarded different responses were given the presence of different kinds of stimuli) rather than monitoring one's own cognitive states. Some researchers (e.g., Hampton, 2009; Inman \& Shettleworth, 1999) have advocated prospective metacognition tasks in which metacognitive responses are available before presenting the test materials, to counter some nonmetacognitive accounts of possible metacognitive performances. 
In a subsequent study, therefore, we investigated pigeons' prospective metamemory during a reference memory task (Iwasaki, Watanabe, \& Fujita, 2018). In training, the subjects learned two rules separately: to discriminate "familiar" and "novel" lists signaled by different colored self-start icons (phase 1), and to familiarize with hint-available and hint-unavailable trials (phase 2). In phase 2, when pigeons responded to a "hint-unavailable trial" icon after pecking a neutral self-start icon, they had to solve normal three-item simultaneous chaining tasks. However, the hint-unavailable trial icon led to a hint that visibly framed the correct item in the proper sequence. After the two phases of training, subjects took a test that combined a hint option with "familiar" and "novel" lists (Figure 2). At first, the self-start icon signaling "familiar" or "novel" lists were presented. After pecking the self-start icon, the subject had to choose either a hint-available or hint-unavailable icon before list items were presented. All correct serial responses in no-hint trials were reinforced by food, but only $60 \%$ or $75 \%$ of correct hint trials were reinforced. We analyzed whether pigeons' hint seeking differed between the highly familiar list and novel lists. We found that two of four pigeons chose the hint trial significantly more often before receiving a novel list than the familiar list. Moreover, one bird did this robustly even in the earliest test sessions. These findings allow us to reject the possibility of response competition or length of delay until the task, because the pigeons decided to seek hints or not before the list items appeared. Also, simply learning to go for a "hint trial" on novel lists to maximize rewards is unlikely, because metacognitive judgment icons were never associated with self-start icons that served as discriminative cues for the type of lists ("familiar" or "novel" lists) in the training phase. Of course, our results do not eliminate all possible nonmetacognitive accounts (e.g., Jozefowiez, Staddon, \& Cerutti, 2009; Le Pelley, 2012; Smith, Beran, Couchman, \& Coutinho, 2008), but they counter the view that pigeons used non-metacognitive or public cues, as proposed by Hampton (2009). Our study suggests that the pigeons could control their hintseeking behavior according to their reference memory or knowledge states.

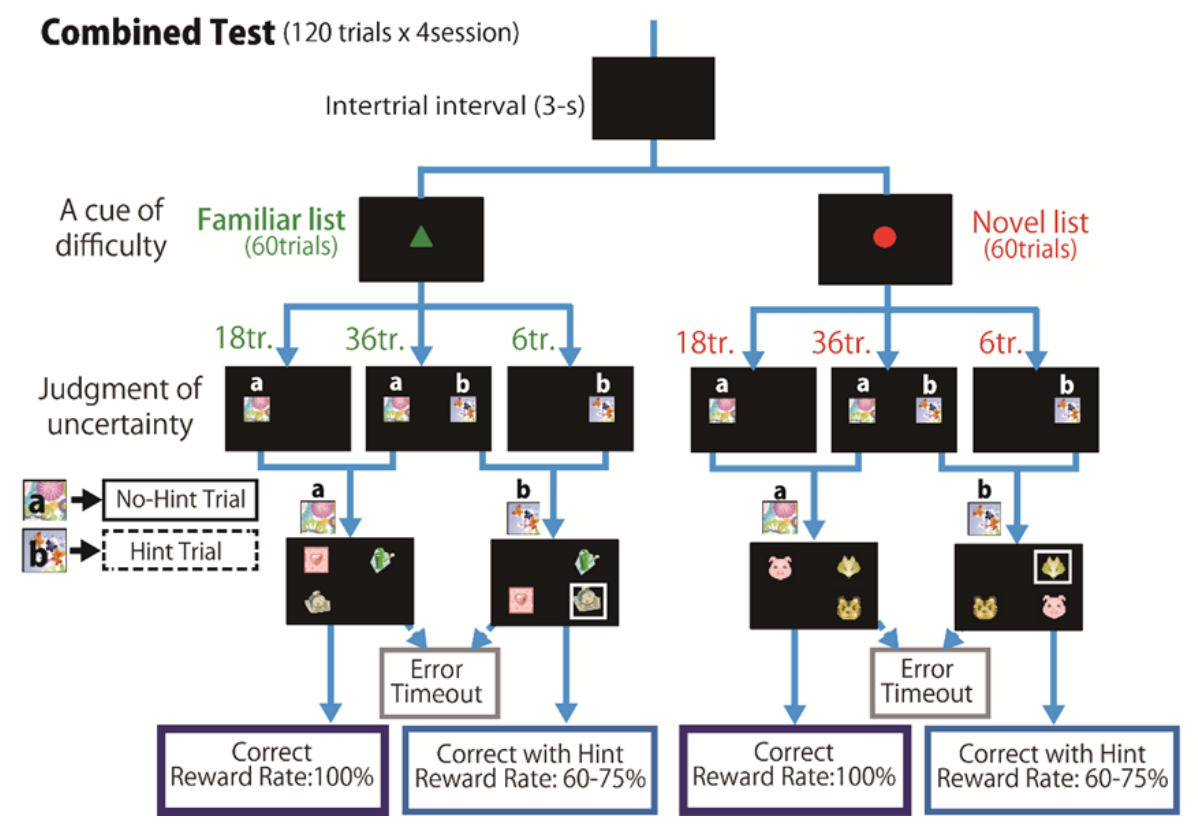

Figure 2. A schematic representation of Iwasaki et al. (2018). In this test, pigeons were presented with a trial that combined rules of two training phases. After ITI, either the green triangle or the red circle appeared as a self-start key. Before proceeding to each associated list, the hint-available icon, the hint-unavailable icon, or both icons were presented. Subjects were able to obtain a hint by responding to the hint-available icon. All correct responses without hints were reinforced by food, but only $60 \%$ or $75 \%$ of correct responses on hint trials were reinforced. Incorrect responses resulted in a timeout irrespective of whether a hint was used. If pigeons were able to judge their uncertainty, they should choose the hint-available icon before solving a task with the novel list. From Iwasaki, S., et al. (2018). Pigeons (Columba livia) know when they will need hints: Prospective metacognition for reference memory? Animal Cognition, 21, 207-217. Copyright 2018 by Springer Nature. 
Although recent studies have provided preliminary evidence of metamemory or metacognition in scrub-jays (Watanabe \& Clayton, 2016; Watanabe, Grodzinski, \& Clayton, 2014), there are relatively few studies of metamemory in birds other than pigeons. Further metamemory studies in avian species that have shown various high-order cognitive abilities (e.g., parrots) would be valuable for building a more complete picture of the relationships between cognitive abilities and metamemory.

We advocate that experiments on metacognition or metamemory in non-human animals need to apply cognitive tasks that are appropriate for the species. In comparison with the strong evidence for metacognition in rhesus monkeys (an Old World primate species), studies of capuchin monkeys (a New World primates) have reported considerably weaker evidence of metacognition (e.g., Basile, Hampton, Suomi, \& Murray, 2009; Beran \& Smith, 2011; Beran, Smith, Coutinho, Couchman, \& Boomer, 2009; Paukner, Anderson, \& Fujita, 2006; Takagi \& Fujita, 2018), with claims for metacognition rare (Fujita, 2009). Beran, Perdue, Church, and Smith (2016) suggested that such differences in results reflect not differences in metacognitive ability, but in risk tolerance. Those authors found that in a greater risk condition capuchin monkeys showed uncertainty responses appropriate to their perceptual states. Again, sound evaluation of metamemory abilities in non-human animals requires the use of suitable cognitive tasks for the target species.

In conclusion, although previous studies using a higher working memory load task or the DMTS task have shown only weak evidence of metamemory in pigeons, we demonstrated metamemory responses during a reference memory task requiring reduced working memory loads. Our studies illustrate the importance of applying suitable cognitive tasks for the target species. As a future research question, we suggest investigating whether differences in metamemory function between humans and pigeons are related to working memory capacity or metamemory function per se. Such work will require a suitable experimental approach and could provide new insights into the mechanisms and perhaps even the evolution of metacognition.

\section{Acknowledgments}

We thank James R. Anderson for his editing of the manuscript. Our studies were supported by the Japan Society for the Promotion of Science (JSPS) Grants-in-Aid for Scientific Research (24-5257 to S. Iwasaki; 20220004, 25118002, 25240020, 16H01505, and 16H06301 to K. Fujita).

\section{References}

Adams, A., \& Santi, A. (2011). Pigeons exhibit higher accuracy for chosen memory tests than for forced memory tests in duration matching-to-sample. Learning and Behavior, 39, 1-11. http://dx.doi.org/10.1007/s13420-0100001-7

Basile, B. M., Hampton, R. R., Suomi, S. J., \& Murray, E. A. (2009). An assessment of memory awareness in tufted capuchin monkeys (Cebus apella). Animal Cognition, 12, 169-180. http://dx.doi.org/10.1007/s10071-008$0180-1$

Beran, M. J., Perdue, B. M., Church, B. A., \& Smith, J. D. (2016). Capuchin monkeys (Cebus apella) modulate their use of an uncertainty response depending on risk. Journal of Experimental Psychology: Animal Learning and Cognition, 42, 32-43. http://dx.doi.org/10.1037/xan0000080

Beran, M. J., \& Smith, J. D. (2011). Information seeking by rhesus monkeys (Macaca mulatta) and capuchin monkeys (Cebus apella). Cognition, 120, 90-105. http://dx.doi.org/10.1016/j.cognition.2011.02.016

Beran, M. J., Smith, J. D., Coutinho, M. V. C., Couchman, J. J., \& Boomer, J. (2009). The psychological organization of "uncertainty" responses and "middle" responses: A dissociation in capuchin monkeys (Cebus apella). Journal of Experimental Psychology. Animal Behavior Processes, 35, 371-381. http://dx.doi.org/10.1037/a0014626

Brown, E. K., Basile, B. M., Templer, V. L., \& Hampton, R. R. (2019). Dissociation of memory signals for metamemory in rhesus monkeys (Macaca mulatta). Animal Cognition, 22, 331-341. http://dx.doi.org/10.1007/s10071-019-01246-5

Call, J., \& Carpenter, M. (2001). Do apes and children know what they have seen? Animal Cognition, 3, 207-220. http://dx.doi.org/10.1007/s100710100078 
Castro, L., \& Wasserman, E. A. (2012). Information-seeking behavior: Exploring metacognitive control in pigeons. Animal Cognition, 16, 241-254. http://dx.doi.org/10.1007/s10071-012-0569-8

Finley, J. R., Benjamin, A. S., \& McCarley, J. S. (2014). Metacognition of multitasking: How well do we predict the costs of divided attention? Journal of Experimental Psychology: Applied, 20, 158-165. http://dx.doi.org/10.1037/xap0000010

Foote, A. L., \& Crystal, J. D. (2007). Metacognition in the rat. Current Biology, 17, 551-555. http://dx.doi.org/10.1016/j.cub.2007.01.061

Fujita, K. (2009). Metamemory in tufted capuchin monkeys (Cebus apella). Animal Cognition, 12, 575-585. http://dx.doi.org/10.1007/s10071-009-0217-0

Goto, K., \& Watanabe, S. (2012). Large-billed crows (Corvus macrorhynchos) have retrospective but not prospective metamemory. Animal Cognition, 15, 27-35. http://dx.doi.org/10.1007/s10071-011-0428-z

Hampton, R. R. (2001). Rhesus monkeys know when they remember. Proceedings of the National Academy of Sciences of the United States of America, 98, 5359-5362. http://dx.doi.org/10.1073/pnas.071600998

Hampton, R. R. (2009). Multiple demonstrations of metacognition in nonhumans: Converging evidence or multiple mechanisms? Comparative Cognition \& Behavior Reviews, 4, 17-28. http://dx.doi.org/10.3819/ccbr.2009.40002

Inman, A., \& Shettleworth, S. J. (1999). Detecting metamemory in nonverbal subjects: A test with pigeons. Journal of Experimental Psychology: Animal Behavior Processes, 25, 389-395. http://dx.doi.org/10.1037/00977403.25.3.389

Iwasaki, S., Watanabe, S., \& Fujita, K. (2013). Do pigeons (Columba livia) seek information when they have insufficient knowledge? Animal Cognition, 16, 211-221. http://dx.doi.org/10.1007/s10071-012-0566-y

Iwasaki, S., Watanabe, S., \& Fujita, K. (2018). Pigeons (Columba livia) know when they will need hints: Prospective metacognition for reference memory? Animal Cognition, 21, 207-217. http://dx.doi.org/10.1007/s10071-017-1153-z

Jozefowiez, J., Staddon, J. E. R., \& Cerutti, D. T. (2009). Reinforcement and metacognition. Comparative Cognition \& Behavior Reviews, 4, 58-60. http://dx.doi.org/10.3819/ccbr.2009.40007

Kornell, N., Son, L., \& Terrace, H. (2007). Transfer of metacognitive skills and hint seeking in monkeys. Psychological Science, 18, 64-71. http://dx.doi.org/10.1111/j.1467-9280.2007.01850.x

Le Pelley, M. E. (2012). Metacognitive monkeys or associative animals? Simple reinforcement learning explains uncertainty in nonhuman animals. Journal of Experimental Psychology: Learning Memory and Cognition, 38, 686-708. http://dx.doi.org/10.1037/a0026478

Maki, W., \& Hegvik, D. (1980). Directed forgetting in pigeons. Animal Learning \& Behavior, 8, 567-574. http://dx.doi.org/10.3758/BF03197771

Nakamura, N., Watanabe, S., Betsuyaku, T., \& Fujita, K. (2011). Do birds (pigeons and bantams) know how confident they are of their perceptual decisions? Animal Cognition, 14, 83-93. http://dx.doi.org/10.1007/s10071-010-0345-6

Nelson, T. O. (1996). Consciousness and metacognition. American Psychologist, 51, 102-116. doi.org/10.1037/0003-066X.51.2.102

Paukner, A., Anderson, J. R., \& Fujita, K. (2006). Redundant food searches by capuchin monkeys (Cebus apella): A failure of metacognition? Animal Cognition, 9, 110-117. http://dx.doi.org/10.1007/s10071-005-0007-2

Sannomiya, M., \& Ohtani, K. (2015). Does a dual-task selectively inhibit the metacognitive activities in text revision? Thinking Skills and Creativity, 17, 25-32. http://dx.doi.org/10.1016/j.tsc.2015.04.002

Smith, J. D., Beran, M. J., Couchman, J. J., \& Coutinho, M. V. C. (2008). The comparative study of metacognition: Sharper paradigms, safer inferences. Psychonomic Bulletin \& Review, 15, 679-691. http://dx.doi.org/10.3758/PBR.15.4.679

Smith, J. D., Couchman, J. J., \& Beran, M. J. (2014). Animal metacognition: A tale of two comparative psychologies. Journal of Comparative Psychology, 128, 115-131. http://dx.doi.org/10.1037/a0033105

Smith, J. D., Coutinho, M. V. C., Church, B., \& Beran, M. J. (2013). Executive-attentional uncertainty responses by rhesus macaques (Macaca mulatta). Journal of Experimental Psychology: General, 142, 458-475. http://dx.doi.org/10.1037/a0029601

Smith, J. D., Shields, W. E., Allendoerfer, K. R., \& Washburn, D. A. (1998). Memory monitoring by animals and humans. Journal of Experimental Psychology: General, 127, 227-250. doi.org/10.1037/0096-3445.127.3.227

Suda-King, C. (2008). Do orangutans (Pongo pygmaeus) know when they do not remember? Animal Cognition, 11, 21-42. http://dx.doi.org/10.1007/s10071-007-0082-7 
Sutton, J. E., \& Shettleworth, S. J. (2008). Memory without awareness: Pigeons do not show metamemory in delayed matching to sample. Journal of Experimental Psychology. Animal Behavior Processes, 34, 266-282. http://dx.doi.org/10.1037/0097-7403.34.2.266

Takagi, S., \& Fujita, K. (2018). Do capuchin monkeys (Sapajus apella) know the contents of memory traces?: A study of metamemory for compound stimuli. Journal of Comparative Psychology, 132, 88-96. http://dx.doi.org/10.1037/com0000087

Templer, V. L., Brown, E. K., \& Hampton, R. R. (2018). Rhesus monkeys metacognitively monitor memories of the order of events. Scientific Reports, 8, 11541. http://dx.doi.org/10.1038/s41598-018-30001-y

Templer, V. L., Lee, K. A., \& Preston, A. J. (2017). Rats know when they remember: Transfer of metacognitive responding across odor-based delayed match-to-sample tests. Animal Cognition, 20, 891-906. http://dx.doi.org/10.1007/s10071-017-1109-3

Terrace, H. S. (1991). Chunking during serial learning by a pigeons I. Basic evidence. Journal of Experimental Psychology: Animal Behavior Processes, 17, 81-93. http://dx.doi.org/10.1037/0097-7403.17.1.81

Watanabe, A., \& Clayton, N. S. (2016). Hint-seeking behaviour of western scrub-jays in a metacognition task. Animal Cognition, 19, 53-64. http://dx.doi.org/10.1007/s10071-015-0912-y

Watanabe, A., Grodzinski, U., \& Clayton, N. S. (2014). Western scrub-jays allocate longer observation time to more valuable information. Animal Cognition, 17, 859-867. http://dx.doi.org/10.1007/s10071-013-0719-7

Wright, A. A., Santiago, H. C., Sands, S. F., Kendrick, D. F., \& Cook, R. G. (1985). Memory processing of serial lists by pigeons, monkeys, and people. Science, 229, 287-289. http://dx.doi.org/10.1126/science.9304205

Yuki, S., \& Okanoya, K. (2017). Rats show adaptive choice in a metacognitive task with high uncertainty. Journal of Experimental Psychology: Animal Learning and Cognition, 43, 109-118. http://dx.doi.org/10.1037/xan0000130 